Check for updates

Cite this: RSC Adv., 2018, 8, 30106

\title{
Expeditious isomerization of glucose to fructose in aqueous media over sodium titanate nanotubes $\uparrow$
}

\author{
Sandeep Kumar, $t^{\text {ac }}$ Devadutta Nepak, $\dot{t}^{\mathrm{b}}$ Sushil Kumar Kansal ${ }^{\mathrm{c}}$ \\ and Sasikumar Elumalai $(\mathbb{D}$ *a
}

Isomerization reaction of glucose to fructose over sodium titanate nanotubes (Na-TNTs) as a Lewis base catalyst was studied. Analytical instruments recorded the specific structural, textural and basic properties of the as-synthesized Na-TNTs. Furthermore, studying the catalytic isomerization performance of the Na-TNTs confirmed their high catalytic efficiency and suitability in aqueous media. The catalyst prompted rapid glucose isomerization within 2 min by achieving nearly half of the maximum yield, whereas with a prolonged reaction up to 15 min the maximum glucose conversion could be reached with $31.26 \%$ fructose yield and $65.26 \%$ selectivity under relatively lower operating conditions $\left(100{ }^{\circ} \mathrm{C}\right.$ and $10 \%$ wt catalyst dose). However, the recyclability performance of the catalyst was not impressive due to the accelerated leaching of cations and surface retention of carbonaceous content, resulting in $\sim 16 \%$ reduced yield after 4 runs. A simple regeneration technique using $\mathrm{NaOH}$ led to the initial catalytic activity being totally regained. Overall, a titania-based catalyst (preferably nanotube structured sodium titanate) was shown as a potential catalyst for large-scale demonstration of glucose isomerization to achieve high fructose productivity.

Received 22nd May 2018

Accepted 20th August 2018

DOI: $10.1039 / \mathrm{c} 8 \mathrm{ra0} 4353 a$

rsc.li/rsc-advances both sugar molecules at each specific reaction temperature. ${ }^{2} \mathrm{On}$ the other hand, the glucose isomerization (GI) reaction is predominantly applied in the food manufacturing sectors, where fructose is used as the primary ingredient in the production of food and beverage items due to its sweeter taste than glucose. Popularly, biochemical (involving glucose isomerase) and chemical (involving $\mathrm{NaOH}$ ) methods are commercially used for this purpose. However, these suffer from serious drawbacks, e.g., low tolerance in variations of feedstock quality, prolonged reaction, product yield $(20-26 \%$ fructose yield, in the case of $\mathrm{NaOH}$ ), undesired side products and odors, toxicity, etc. ${ }^{3}$ With the interest of production of HMF from glucose, the majority of studies have suggested using a multi-step protocol owing to the advantages that fructose could be more easily, quickly, and completely dehydrated to obtain the target compound than glucose due to its higher reactivity., ${ }^{2,-6}$

In this context, a heterogeneous catalytic approach is preferred owing to the higher selectivity and yield, operation under a wide range of conditions, minimal side product generation, facility of recovery and regeneration, and environmentally safe process. ${ }^{7}$ To date, the selective glucose to fructose conversion has been demonstrated using a different category of solid materials, including modified zeolites, ${ }^{8-10}$ metal oxides, ${ }^{11}$ hydrotalcites, ${ }^{12}$ and metallosilicates. ${ }^{13}$ Therefore, GI reaction is possible through Lewis acid and/or Lewis base catalysis, however, each of the protocols undergo dissimilar reaction pathways resulting in delivering varied final product concentrations. In perception, Lewis base catalysis is promising over 
the other due to better selectivity and high yield, and offers less chance of further transformation of formed fructose to HMF in aqueous media., ${ }^{2,14}$ Moreover, it can be handled in ambient conditions because the adsorption of carbon dioxide or water is not too strong that considerably reduces the product yield. ${ }^{15}$ In recent years, efforts are being made to develop ideal catalyst materials through a variety of modified approaches to overcome the drawbacks facing during large-scale processing, i.e., catalyst instability (activity loss) and less recycle ability, and higher expenses at catalyst preparation. For instance, Faria et al. developed a catalyst by growing carbon nanotubes on $\mathrm{NaX}$ faujasite zeolites with the interest of converting glucose to HMF. The Lewis base catalysis could achieve as high as $26 \mathrm{~mol} \mathrm{~kg}^{-1}$ $\mathrm{h}^{-1}$ fructose formation (with $85 \%$ selectivity when glucose conversion is $<20 \%$ ) in aqueous medium at $110{ }^{\circ} \mathrm{C}$. Fascinatingly, the catalyst offered improved hydrothermal stability and resistance against leaching of the active sites. ${ }^{16}$ Indeed, the specific surface area of catalyst promotes the catalytic activity of reaction. At the same time, the enhancement of the external surface area harmfully contributes to the possible diffusion of molecules. ${ }^{17}$

Among the classified low-cost metal oxide catalysts, titania (or titanium dioxide, $\mathrm{TiO}_{2}$ ) is proven to be stable under rigorous reaction conditions, offer excellent surface area, good ion exchangeability, better recyclable performance, inert and safe material. Whereas, nanotubes possess high specific surface area and hollow morphology and therefore, it is widely researched in many areas, including chemical catalysis, photocatalysis, electrocatalysis, sensors, and lithium batteries compared to other regular forms (nanoribbon, nanowire, nanosheet). ${ }^{17,18}$ Moreover, the characteristic $\mathrm{TiO}_{2}$ exhibit a bifunctional property, i.e., Lewis acid and base attributed to the lattice $\mathrm{O}^{-}$and $\mathrm{Ti}^{+}$ions, and therefore, it has been extensively applied for chemical reactions. ${ }^{\mathbf{1 9 , 2 0}}$ For instance, Lanziano et al. demonstrated the catalytic performance of $\mathrm{TiO}_{2}$ (superficial area of $128 \mathrm{~m}^{2} \mathrm{~g}^{-1}$ ) on glucose to fructose conversion in aqueous medium, but only $12 \%$ wt fructose yield could be obtained at $120{ }^{\circ} \mathrm{C}$ claiming limited catalytic activity. ${ }^{21}$ Therefore, in the present study, titanate nanotube consisting $\mathrm{Na}^{+}$as charge compensating cation exhibiting a Lewis base character was invested for the GI reaction in aqueous medium with the objective of demonstrating a feasible, cost-effective process method. ${ }^{18}$ For this purpose, $\mathrm{Na}^{+}$is the most investigated candidate due to its greater affinity towards the support material, more active, easy availability and cheap compared to other common monovalent (commonly, $\mathrm{Li}^{+}, \mathrm{Na}^{+}, \mathrm{K}^{+}, \mathrm{Cs}^{+}$) and divalent $\left(\mathrm{Ca}^{++}\right.$and $\left.\mathrm{Ba}^{++}\right)$cations. ${ }^{22}$ In hypothesis, the sodium titanate $\left(\mathrm{Na}_{2} \mathrm{Ti}_{3} \mathrm{O}_{7}\right)$ offers large basic sites due to the presence of two lone pair electrons $\left(\mathrm{O}^{-}\right)$that allows more ion compensation is responsible for the basicity, thereby promoting the isomerization reaction.

\section{Materials and methods}

\section{Chemicals}

All chemicals and reagents $\left(\mathrm{TiO}_{2}\right.$ anatase, $\mathrm{NaOH}, \mathrm{HCl}, \mathrm{D}^{-}$ glucose, D-fructose, and D-mannose, ethanol, methanol, 1- propanol, $\mathrm{LiNO}_{3}$ of $99 \%$ purity, $\mathrm{KNO}_{3}$ of $99 \%$ purity, and $\mathrm{CsNO}_{3}$ of $99 \%$ purity) were purchased from Sigma Aldrich India as analytical grade and used without any modification.

\section{Sodium titanate nanotube synthesis}

Sodium titanate nanotubes (Na-TNT) was prepared by conventional hydrothermal synthesis method. ${ }^{23,24}$ Briefly, $1.0 \mathrm{~g} \mathrm{TiO}_{2}$ powder was added to $80 \mathrm{ml}$ of $10 \mathrm{M} \mathrm{NaOH}$ solution in $100 \mathrm{ml}$ beaker. The mixture was continuously stirred for $8 \mathrm{~h}$ at $200 \mathrm{rpm}$ to achieve a homogeneous mix, transferred to a Teflon lined stainless steel autoclave, and heated for 5 days at $180{ }^{\circ} \mathrm{C}$. The resultant solids were collected and washed using ethanol-water mixture (50: 50 ratio) until the $\mathrm{pH}$ becomes neutral. The assynthesized catalyst was dried at $80{ }^{\circ} \mathrm{C}$ overnight. Other titanate nanotube catalysts $\mathrm{x}$-TNT, where $\mathrm{x}=\mathrm{Li}^{+}, \mathrm{K}^{+}$, and $\mathrm{Cs}^{+}$were prepared from Na-TNT through ion exchange. About $100 \mathrm{mg} \mathrm{Na}$ TNT was added to the corresponding nitrate solution of $0.5 \mathrm{M}$ conc. (prepared in milli Q water) and stirred continuously for $24 \mathrm{~h}$ (at $200 \mathrm{rpm}$ ) at $80{ }^{\circ} \mathrm{C}$. The slurry mixture was washed repeatedly using milli $\mathrm{Q}$ water and the solids collected were dried overnight at $50{ }^{\circ} \mathrm{C}$. Similarly, the $\mathrm{H}_{2} \mathrm{Ti}_{3} \mathrm{O}_{7}$ catalyst was prepared by using $1 \mathrm{M} \mathrm{HCl}$ solution, as reported elsewhere. ${ }^{17}$

\section{Characterization techniques}

Powder X-ray diffraction (XRD) analysis was performed to the as-synthesized Na-TNT on Philips $\mathrm{X}^{\prime}$ Pert Pro Super X-ray diffractometer $\mathrm{Cu}-\mathrm{K} \alpha$ radiation $(\lambda=1.54056 \AA)$ operated at 40 $\mathrm{kV}$ and $30 \mathrm{~mA}$. The structural morphology and chemical composition were determined by SEM-EDX equipment (JEOL 5900 LV microscope). The size and morphology were measured by using a high-resolution transmission electron microscopy (HRTEM) images and selected-area electron diffraction (SAED) using JEOL 2010 microscope (resolving power $1.9 \AA$ At $200 \mathrm{kV}$ ). The nitrogen adsorption-desorption isotherms were obtained using a Micromeritics ASAP 2020 automatic analyzer at $(-80$ $\left.{ }^{\circ} \mathrm{C}\right)$. Before analysis, the samples were degassed at less than $10^{-1} \mathrm{~Pa}$ pressure at $250{ }^{\circ} \mathrm{C}$ for $6 \mathrm{~h}$. BET method was used to calculate the specific surface area (SBET), and the total pore volume $\left(V_{\mathrm{p}}\right)$ was determined by nitrogen adsorption capacity at a relative pressure of 0.98 . Similarly, the average pore diameter (DP) and pore size distributions were obtained from the adsorption isotherms by the $\mathrm{BJH}$ method. Temperatureprogrammed desorption of adsorbed $\mathrm{CO}_{2}\left(\mathrm{CO}_{2}\right.$-TPD) technique was used to measure the basic sites of Na-TNT. Briefly, $0.1 \mathrm{~g}$ of catalyst was placed in a fixed-bed U-shaped quartz reactor. The sample was first pretreated in He flow (30 $\mathrm{ml} \min ^{-1}$ ) at $350{ }^{\circ} \mathrm{C}$ for $2 \mathrm{~h}$, cooled down at room temperature, and flushed with a $\mathrm{CO}_{2} / \mathrm{He}$ gas mixture $\left(50 \%\right.$ at $30 \mathrm{ml} \mathrm{min} \mathrm{m}^{-1}$ flow) for $30 \mathrm{~min}$. Followed by, purging of the remaining $\mathrm{CO}_{2}$ with He flow. The TPD was run from temperature 100 to $600{ }^{\circ} \mathrm{C}$ at a constant heating rate $\left(10{ }^{\circ} \mathrm{C} \mathrm{min}^{-1}\right.$ in He gas stream). The basicity of the catalyst was estimated as being the total amount of $\mathrm{CO}_{2}$ released through thermal programmed desorption per gram of the Na-TNT sample. Na to Ti atomic ratio of the catalyst was determined by ICP-AES analysis (Shimadzu, ICP-1000IV). 


\section{Glucose isomerization to fructose}

Glucose isomerization reaction was carried out in a $3 \mathrm{ml}$ thickwalled glass reactor. In a typical reaction, glucose $1 \mathrm{ml}$ of $1.5 \%$ stock solution) and the as-synthesized catalyst were loaded into the reactor (maximum $16.67 \%$ wt on glucose) and sealed tightly. The reactor was pre-warmed depending on the reaction temperature and heated up to $120^{\circ} \mathrm{C}$ in an oil-bath using a hot plate stirrer at $200 \mathrm{rpm}$. Once the reaction is completed (maximum $5 \mathrm{~h}$ ), the reactor was cooled down immediately in an ice bath. The catalyst particles were filtered off by passing through $0.45 \mu \mathrm{m}$ membrane under vacuum condition (S1). The filtrate collected was analyzed by high-performance liquid chromatography (HPLC, Agilent Technologies 1200 infinity series) after requisite dilution. The HPLC system was equipped with Agilent Hi-Plex Ca column (300 mm length, $8 \mu \mathrm{m}$ porosity), and maintained at $85{ }^{\circ} \mathrm{C}$ and $0.6 \mathrm{ml} \mathrm{min}{ }^{-1}$ of HPLC grade water (mobile eluent). The carbohydrates were analyzed on UV detector operated at $50{ }^{\circ} \mathrm{C}$. The concentration of sugars was estimated using their respective calibration charts prepared by commercial grade standards. The glucose and products yield (fructose and mannose) was calculated based on the difference in concentration of residual glucose and products to initial glucose concentration, respectively. Similarly, the products selectivity were calculated based on the difference in concentration of products to glucose reacted. All experiments were conducted in triplicate and the data reported is the average.

\section{Catalyst recovery and regeneration}

The collected catalyst solid after the first run (S1) was appeared to be slightly yellowish in color was washed repeatedly using milli-Q water until it becomes natural white (S2). It was dried at room temperature and freeze-dried for up to $48 \mathrm{~h}$. The same procedure was repeated for each recycle runs. The catalyst regeneration was performed to the solid materials collected after four recycle runs. It was calcined at $300{ }^{\circ} \mathrm{C}$ before treating with $2 \mathrm{M} \mathrm{NaOH}$ solution in $100 \mathrm{ml}$ beaker. The mixture was heated for $12 \mathrm{~h}$ at $80{ }^{\circ} \mathrm{C}$ under continuous stirring condition (200 rpm). The resultant solid material was collected and washed repeatedly using an ethanol-water mixture (50:50 ratio) until the $\mathrm{pH}$ becomes neutral.

\section{Results and discussion}

\section{Characterization of as-synthesized sodium titanate nanotubes}

Of the classical preparation of methods of titanate nanotube (TNT) using $\mathrm{TiO}_{2}$ as a precursor, hydrothermal treatment with alkali is of particular interest because nanotube aggregates can be produced in an unsophisticated manner at low temperatures. Indeed, the characteristic $\mathrm{TiO}_{2}$ in the form of nanotubes have a relatively larger specific surface area $\left(400 \mathrm{~m}^{2} \mathrm{~g}^{-1}\right)$ and pore diameter (5-7 nm inner and 7-11 nm outer diameter) than that in the form of nanoparticles $\left(128 \mathrm{~m}^{2} \mathrm{~g}^{-1}\right) .{ }^{21,25}$ Results in providing larger availability of surface active sites and facilitate interactions with the support which then can lead to a higher activity of the catalyst particles. ${ }^{23,26}$ Moreover, the nanotubes have a distinctive ion-exchangeable ability due to their layered structure and permit mobility of $\mathrm{Na}^{+}$and $\mathrm{H}^{+}$in the interlayer of TNTs. Owing to these advantages, the synthesis of titanate nanotubes became a routine, and it is generally accepted that the nanotubes have a layered titanate structure. However, there is a discrepancy in the proposed models of formation of titanate nanotubes in the hydrothermal environment and also, between the crystal structure and chemical composition. Furthermore, the stability and other structural characteristics, including pore structure and crystallinity (the common crystalline habits are anatase, rutile, and brookite, which own different band gap energies and chemical activities) of the $\mathrm{TiO}_{2}$ nanotube are dependent on the preparation method and subsequent calcination temperatures. ${ }^{25,27}$ Therefore, the as-synthesized sodium titanate nanotube (Na-TNT) obtained using $\mathrm{TiO}_{2}$ anatase, as a precursor, through hydrothermal treatment in $\mathrm{NaOH}$ solution at $180{ }^{\circ} \mathrm{C}$ was subjected to various instrumental techniques to determine its structural characteristics. The initial chemical analysis of the ion-exchanged TNT revealed that it allowed $c a$. $>60 \%$ ion exchange for $\mathrm{Na}^{+}$ions. Further, the instrumental analysis of the nanotubes by ICP-OES verified the atomic ratio of $\mathrm{Na}$ to $\mathrm{Ti}$ as 0.65 , which lies in the close range to the theoretical value of $\mathrm{Na}_{2} \mathrm{Ti}_{3} \mathrm{O}_{7}(0.67)$. Presumably, it is caused due to some of the $\mathrm{Na}^{+}$ions were exchanged by $\mathrm{H}^{+}$during the $\mathrm{H}_{2} \mathrm{O}$ washing treatment, which leads to the synthesis of hydrous sodium titanate of composition $\mathrm{Na}_{x} \mathrm{H}_{2}-x \mathrm{Ti}_{3} \mathrm{O}_{7} \cdot n \mathrm{H}_{2} \mathrm{O}$, resulting in forms multi-walled scroll-type nanotubes. Also, traces of unreacted $\mathrm{TiO}_{2}$ precursor were presented in the synthesized material. ${ }^{28}$

The textural characteristics, mainly BET specific surface area, pore volume, and pore diameter, were determined from $\mathrm{N}_{2}$ adsorption isotherms, as displayed in Fig. 1a. The type IV $\mathrm{N}_{2}$ isotherms with $\mathrm{N}_{2}$ hysteresis loop indicated that solid material is mesoporous and exhibited $155.3 \mathrm{~m}^{2} \mathrm{~g}^{-1}, 0.58 \mathrm{~cm}^{3} \mathrm{~g}^{-1}$, and $7.2 \mathrm{~nm}$, respectively. The XRD pattern (Fig. S1a in the ESI $\dagger$ ) of the solids showed that typical peaks at 10.1, 24.3, 28.4, and $48.5^{\circ}$ corresponding to the reflections from (200), (110), (211) and (020) planes, respectively, of Na-TNT $\left(\mathrm{Na}_{2} \mathrm{Ti}_{3} \mathrm{O}_{7}\right)$. The composition was confirmed using JCPDS-ICDD card 31-1329. The distinct peak at $10.1^{\circ}(2 \theta)$ is attributed to the interlayer distance of Na-TNT, which is equal to $0.80 \mathrm{~nm} .{ }^{25}$ In understanding, this characteristic peak and its position confirm the presence of $\mathrm{Na}$ in the interlayer spaces of the sodium titanate structure. Moreover, the diffraction signals point out the poor crystalline nature of the synthesized nanotubes based on the broadness of all observed peaks. Most importantly, no signals attributable to any crystalline phase of $\mathrm{TiO}_{2}$ or to other types of Na-TNTs (general formula of $\mathrm{Na}_{2} \mathrm{Ti}_{n} \mathrm{O}_{2 n+1}$, where $n=4,5,6$ varies depending on the heat treatment and condition) were observed in the diffraction profile of the synthesized nanotubes. Thus, the analysis result confirms that the successful transformation of $\mathrm{TiO}_{2}$ anatase into Na-TNT through the alkaline hydrothermal synthesis. Representative HR-TEM imaging was acquired to the nanotubes (Fig. 1b), which characterized that solids are in tubular morphology (open-ended tubes with 3 to 5 layered walls and not symmetric) with outer and inner diameters being 9.8 and $4.8 \mathrm{~nm}$, respectively, and of varying lengths ranging up to several tens $\mathrm{nm}$. Though the synthesized material appeared to be tubes are joined together in bundles, the selected area 

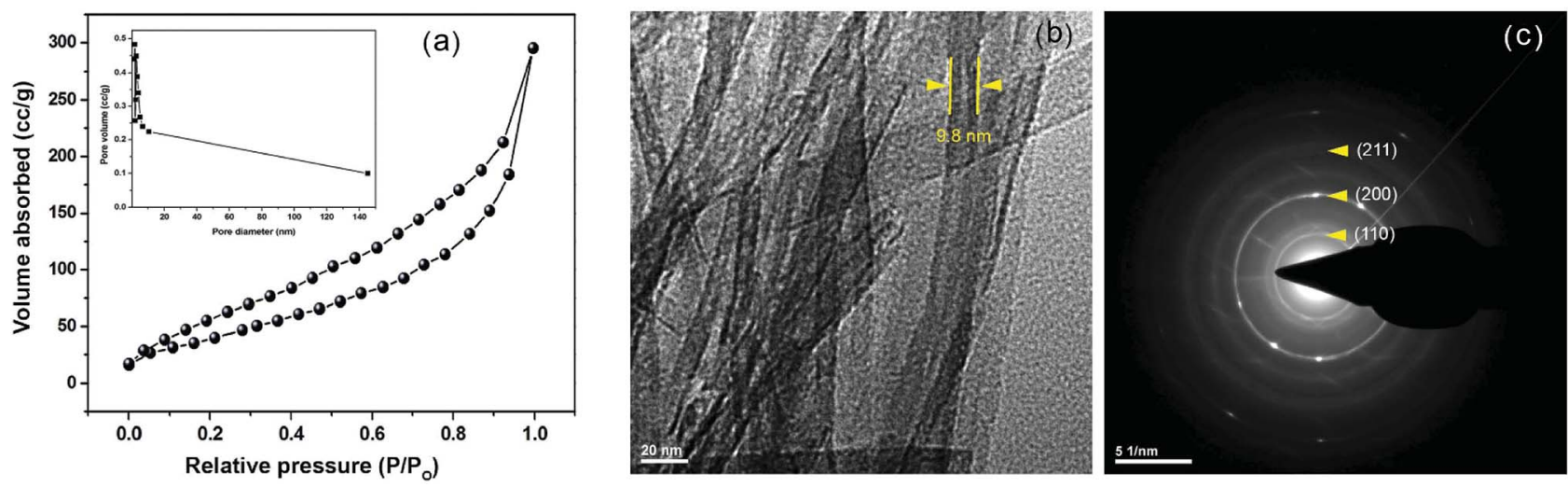

Fig. 1 (a) Typical $\mathrm{N}_{2}$ adsorption-desorption isotherm and BJH pore size distribution plot (inset) of as-synthesized Na-TNT, (b) HR-TEM, and (c) SAED characterization analyses result of the as-synthesized Na-TNT.

electron diffraction (SAED) pattern was recorded using large selected area aperture (Fig. 1c). The analysis results are revealing that the innermost ring to a $d$-spacing of (110) planes, whereas the other two circles to (200) and (211) inter-planer distances, confirming the tube-shaped crystals of Na-TNT. Further, the obtained spectroscopic and structural data were correlated with the morphology on scanning electron microscopy (SEM) with EDX measurement. The imaging result indexed the evident transformation of spherical particles into the fibrous tubular material (Fig. S1b in the ESI $\dagger$ ), whereas the EDX data also reproduced its maximum ion exchange degree $(\sim 65 \%)$.

With regard to the basicity, typical $\mathrm{CO}_{2}$ desorption profile was obtained for the as-synthesized Na-TNT sample through $\mathrm{CO}_{2}$-TDP desorption analysis technique. Results presented (Fig. S2a in the ESI†) that an intermediate signal with the maximum at $\sim 202{ }^{\circ} \mathrm{C}$ corresponds to desorption of $\mathrm{CO}_{2}$ from sites of medium basic strength $\left(106 \mu \mathrm{mol} \mathrm{g}^{-1}\right)$. Whereas, the other two intensity peaks obtained at temperatures $338^{\circ} \mathrm{C}$ and $518{ }^{\circ} \mathrm{C}$ were related to the $\mathrm{CO}_{2}$ desorption from sites with medium $\left(81 \mu \mathrm{mol} \mathrm{g}{ }^{-1}\right)$ and strong basicity $\left(43 \mu \mathrm{mol} \mathrm{g}^{-1}\right)$, respectively. The estimated total amount of basic sites was 231 $\mu \mathrm{mol} \mathrm{g}^{-1}$. However, it is comparatively lower than other conventional Lewis base catalysts, for example, $\mathrm{MgO}$ with 346 $\mu \mathrm{mol} \mathrm{g}{ }^{-1} \cdot{ }^{29}$ Results revealed that nanotube catalysts consisted predominantly weak and medium basic sites. The functional groups of Na-TNT were examined on FTIR spectroscopy between 400 and $4000 \mathrm{~cm}^{-1}$. It can be noticed that nanotubes exhibit a broad and intense band located at about $3300 \mathrm{~cm}^{-1}$ (Fig. S2b in the ESI $\dagger$ ), which is attributed to the $\mathrm{O}-\mathrm{H}$ stretching mode, indicating the presence of surface hydroxyl groups and water molecules adsorbed on the surface and in the interlayer space of the nanotubes. Water molecules were confirmed by the presence of the band at $1630 \mathrm{~cm}^{-1}$ that is assigned to the $\mathrm{H}-\mathrm{O}-\mathrm{H}$ deformation mode. The band observed at $897 \mathrm{~cm}^{-1}$ can be attributed to the Ti-O stretching mode which involves non-bridging oxygen atoms. The band at $470 \mathrm{~cm}^{-1}$ is related to $\mathrm{Ti}-\mathrm{O}-\mathrm{Ti}$ vibrations of the interconnected octahedra that are rigid units responsible for the formation of the nanotube walls. Overall, the analyses result authenticated the expected structural property of the as-synthesized Na-TNT and found to be consistent with the literature reports..$^{25,30,31}$

\section{Performance of Na-TNT on glucose to fructose isomerization}

Typical base catalyzed GI reaction follows the classical Lobry de Bruyn-Alberda van Ekenstein (LdB-AvE) rearrangement mechanism. ${ }^{32}$ Accordingly, the postulated reaction mechanism scheme of the GI reaction in the presence of Na-TNT is depicted in Fig. 2. When the glucose is in contact with $\mathrm{Na}_{2} \mathrm{Ti}_{3} \mathrm{O}_{7}$, the ring opening is induced by the $\mathrm{Na}^{+}$ion, which is the most important step in the isomerization pathway, based on the fact that glucose primarily exists in its ring-form in $\mathrm{H}_{2} \mathrm{O}$ to a greater extent. Further, the hydride ion shift is occurred by the basic sites on the catalyst, the glucose C-2 is deprotonated, resulting in the formation of 1,2-enediol intermediate (trans-enediol). Followed by, a proton is then transferred from C-2 to C- 1 and O2 to $\mathrm{O}-1$ to form fructose, and also, the formation of mannose via epimerization. Alongside, more complex reaction pathways (non-reversible) involving the 2,3-enediol anion, aldolization/ retro-aldolization, $\beta$-elimination, and benzylic rearrangement generates a variety of unwanted byproducts (such as $p$-saccharinic acid, glyceric acid, 3-hydroxypropanoic acid, 3-hydroxypropanol, glyceraldehyde and other compounds). ${ }^{33}$ In concurrence to the base catalyzed isomerization reactions in the presence of $\mathrm{NaOH}$ or organic amines that systematically $\mathrm{Na}^{+}$ion helps in the glucose ring opening mechanism, whereas $\mathrm{OH}^{-}$ion is responsible for the isomerization.

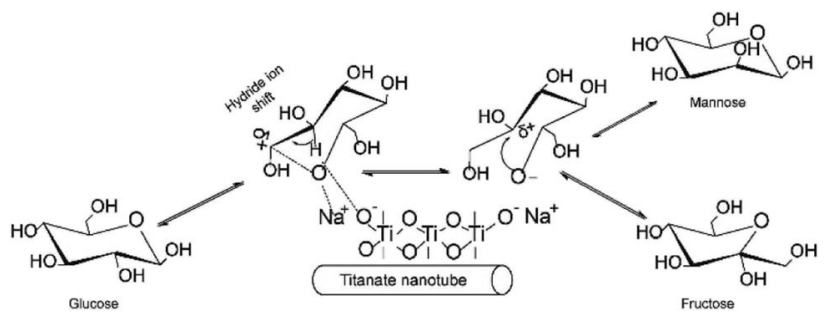

Fig. 2 Proposed reaction scheme of glucose to fructose isomerization over $\mathrm{Na}_{2} \mathrm{Ti}_{3} \mathrm{O}_{7}$. 
Table 1 Response result of glucose isomerization to fructose under varied severity conditions ${ }^{a}$

\begin{tabular}{lllllll}
\hline $\begin{array}{l}\text { Catalyst loading\% wt } \\
\text { per wt Glu }\end{array}$ & $Y_{\text {Fru }}(\%)$ & $S_{\text {Fru }}(\%)$ & $Y_{\text {Man }}(\%)$ & $S_{\text {Man }}(\%)$ & TOF $\left(\times 10^{-3}\right)$ & Carbon balance $(\%)$ \\
\hline 3.33 & 22.75 & 80.28 & 3.27 & 11.54 & 1.49 & 94.41 \\
6.67 & 28.00 & 76.64 & 4.31 & 11.81 & 1.84 & 91.47 \\
10.00 & 30.78 & 68.79 & 5.39 & 12.05 & 2.02 & 86.03 \\
13.33 & 30.21 & 65.59 & 5.76 & 12.50 & 1.98 & 74.15 \\
16.67 & 28.79 & 62.99 & 4.77 & 10.68 & 1.89 & 74.77
\end{tabular}

${ }^{a}$ Reaction conditions: $90^{\circ} \mathrm{C}$ for $30 \mathrm{~min}$ in $\mathrm{H}_{2} \mathrm{O}$ medium. TOF - turn over frequency is calculated as moles of fructose formed per mole of Na charge compensating cation supply per second. Carbon balance is derived based on the difference between moles of carbon in products (fructose and mannose) and unreacted glucose, and to the mole of carbon in the initial glucose. N.D. - not detected. $Y_{\text {Fru }}$-fructose yield; $S_{\text {Fru }}$-fructose selectivity; $Y_{\mathrm{Man}}$-mannose yield; $S_{\mathrm{Man}}$-mannose selectivity.

The rate and extent of these undesired side reactions could be controlled by tuning of reaction parameters, including the type of base cation and its concentration, and temperature. ${ }^{34}$ Consequently, the as-synthesized Na-TNT was tested for its catalytic performance over aldose to ketose isomerization reaction under varying reaction conditions. Initially, the effect of catalyst dose to glucose was investigated under different loading levels ranging from 3.33 to $16.67 \% \mathrm{wt}$, while keeping the other parameters constant (at $90{ }^{\circ} \mathrm{C}$ for $30 \mathrm{~min}$ ). Typical isomerization result was achieved during the incremented loadings up to the optimum, and further continuous supply of catalyst adversely affected the yield (Table 1). For example, a linearly increasing trend of fructose yield with $28 \%$ relative increments up to $10 \%$ wt catalyst load was achieved and started gradually decreasing as the load increases further and reaching as low as relatively $6.46 \%$ at the maximum load condition. Whereas, the fructose selectivity maintained a decreasing fashion from the start of the reaction (from 80.27 to $62.98 \%$ ) even at low catalyst dosage mainly attributed to the further conversion of fructose. That is isomerization to glucose and undergo secondary transformations when it remains longer inside the nanotube structure because of partial blockage by the pores and interaction with the alkali ions present in the nanotubes. $^{35}$ However, the glucose conversion showed a contrary trend with the corresponding incremented catalyst dose (increased from 28.34 to 46.71), this clearly indicates that additional catalyst load, in turn, provides higher basic conditions led to the formation of by-products under harsh conditions $\left(90{ }^{\circ} \mathrm{C}\right.$ for $\left.30 \mathrm{~min}\right)$. Normally, the monosaccharides are unstable under strongly alkaline conditions and led to degrading them into numerous different byproducts at least 50 unwanted compounds. ${ }^{33,34}$ The evident color changes of the medium from pale yellow to dark brown as with the increased catalyst dose at $100{ }^{\circ} \mathrm{C}$ (Fig. S3 in the ESI $\dagger$ ) indicates the progression of this thermally-induced nasty side reactions unconditionally.

Mannose is the other main by product generated during typical GI reaction, formed in parallel to fructose by following the same trend throughout the incremented severities; it accounted for $5.76 \% \mathrm{wt}$ (maximum) at the optimum (10.0\% wt catalyst dose). While correlating the results with the estimated turnover frequency (TOF, moles of fructose produced per mole of charge compensating $\mathrm{Na}$ ion per s, assuming complete ionic compensation exist between $\mathrm{Na}^{+}$and $\mathrm{O}^{-}$) of the reaction data, representing the same bell-shaped trend with maxima depending on the reaction condition (as summarized in Table 1). Furthermore, the results suggested that reaction was catalyzed only on Lewis basic sites without the aid of any of the other properties of support material except textural influence, based on the control run conducted in the presence of $\mathrm{H}_{2} \mathrm{Ti}_{3} \mathrm{O}_{7}$. In which, no fructose was formed due to compensation of ionic charges by $\mathrm{H}^{+}$and $\mathrm{O}^{-}$exhibiting neither base nor acid sites (Fig. S4 in the ESI $\dagger$ ). However, the decreased carbon balance $(92 \%)$ attributed to the sugar degradation at $100{ }^{\circ} \mathrm{C}$. In consistent with the hypothesis that the characteristic $\mathrm{Na}_{2} \mathrm{Ti}_{3} \mathrm{O}_{7}$ possess a Lewis basic site, which plays an important role in determining the catalytic activity of the reaction, suggesting the good ion exchange degree of $\mathrm{Na}^{+}$to be responsible for the basic sites. The obvious decreasing value of carbon balance indicated that significant loss of carbohydrate molecules with the corresponding incremented severities (up to $\sim 33 \%$ with averagely $10 \%$ point differences). The reaction rate can also slow down at higher catalyst dose because of the approach to the thermodynamic equilibrium conversion for the glucose to fructose at the maintained temperature $\left(100{ }^{\circ} \mathrm{C}\right)$. Therefore, the optimum NaTNT/glucose ratio is considered to be $1: 10$, corresponding to a fructose yield and selectivity of $30.80 \%$ and $68.80 \%$, respectively.

In a typical heterogeneous catalysis reaction, the catalytic activity of the reaction is greatly influenced by temperature. With regard to the texture of the support, the characteristic titanate nanotubes exhibit remarkable catalytic performance for the reaction even at room temperature condition compared to the other conventional catalysts. Overall literature studies have shown that relatively low reaction temperatures and times favor aldose isomerization activity under alkaline $\mathrm{pH} .{ }^{33,34}$ In addition, the GI reaction is slightly endothermic. ${ }^{14}$ Therefore, it was expected to achieve a better glucose conversion accompanied with fructose selectivity under low-temperature conditions in the presence of Na-TNT. Consequently, the response of the glucose isomerization reaction was recorded with the correspondingly raised temperatures ranging from room temperature $\left(25^{\circ} \mathrm{C}\right)$ to $120^{\circ} \mathrm{C}$, while maintaining the catalyst to glucose ratio as $1: 10$ for $30 \mathrm{~min}$ constant (Fig. 3). No or less improvement in GI reaction was observed at $25{ }^{\circ} \mathrm{C}(<1 \%$ fructose yield with $28 \%$ glucose conversion), whereas the raised temperatures 


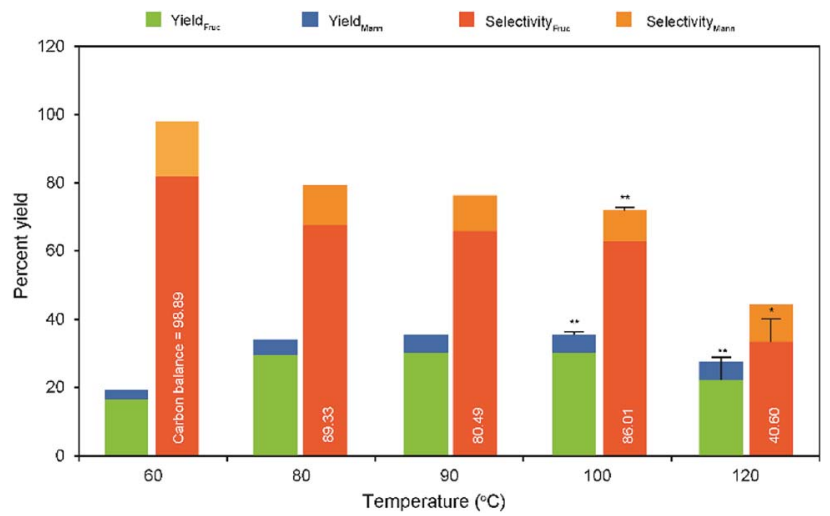

Fig. 3 Effect of temperature on glucose isomerization to fructose in aqueous medium ( $10 \%$ wt catalyst dose on glucose). Error bar represents standard deviation, and asterisks $(*)$ and $(* *)$ indicate significant and non-significant at $p$-value $\leq 0.05$, respectively.

significantly influence products yield. For instance, $\sim 2$-fold relative increase in the fructose yield was obtained when the temperature was raised from 60 to $80^{\circ} \mathrm{C}$ and further increased temperatures showed a slight increment in the yield up to $100{ }^{\circ} \mathrm{C}$, at which a maximum yield was achieved $(30.94 \%$ fructose yield with $62.88 \%$ selectivity). A drastically decreased yield result was recorded beyond the optimum level, i.e., relatively $\sim 27 \%$ under higher temperature conditions, demonstrating higher temperatures favor more sugar degradation reactions. ${ }^{34}$ Whereas, the fructose selectivity was appeared to be the same pattern of decreasing value (relatively as low as $\sim 59 \%$ ) and worse under harsh conditions ( $\sim 2$-fold lower selectivity at 120 $\left.{ }^{\circ} \mathrm{C}\right)$. Although the prolonged reaction times ( $>45 \mathrm{~min}$ ) and at relatively higher temperatures $\left(>60{ }^{\circ} \mathrm{C}\right)$ resulted in higher glucose conversion (ratio of glucose reacted to initial supply), lower fructose yield and selectivity was achieved attributed to the formation of unwanted byproducts through undesired reactions. ${ }^{11,34}$ This was supported by the color change indications of the reaction medium with respect to the temperatures (Fig. S5 in the ESI $\dagger$ ). Mannose exhibited a nearly same production pattern of fructose concentration during the increased temperatures.

\section{Kinetics of glucose isomerization at different temperatures}

Further investigation of the effect of time on GI reaction was studied at different temperatures viz. 60,80 and $100{ }^{\circ} \mathrm{C}$. The comparative results show that the reaction is time dependent and catalytic activity had a significant impact on the influence of the reaction (Fig. 4). For example, nearly $65 \%$ of the maximum yield (i.e., $31.26 \% \mathrm{wt}$ after $15 \mathrm{~min}$ ) could be reached within 2 min reaction at $100{ }^{\circ} \mathrm{C}$, whereas the other reactions conducted at lower temperatures took $30 \mathrm{~min}$ (maximum) to reach the marked level. Further prolonged reaction attained the maximum fructose concentration at varied times depending on the temperature, e.g., $31.26 \%$ with $65.26 \%$ fructose selectivity within $15 \mathrm{~min}$ at $100{ }^{\circ} \mathrm{C}$. Whereas, the low temperature reactions at $80{ }^{\circ} \mathrm{C}$ and $60{ }^{\circ} \mathrm{C}$ could yield the maximum after $30 \mathrm{~min}$ (30.31\% with $67.60 \%$ fructose selectivity) and $150 \mathrm{~min}(28.80 \%$
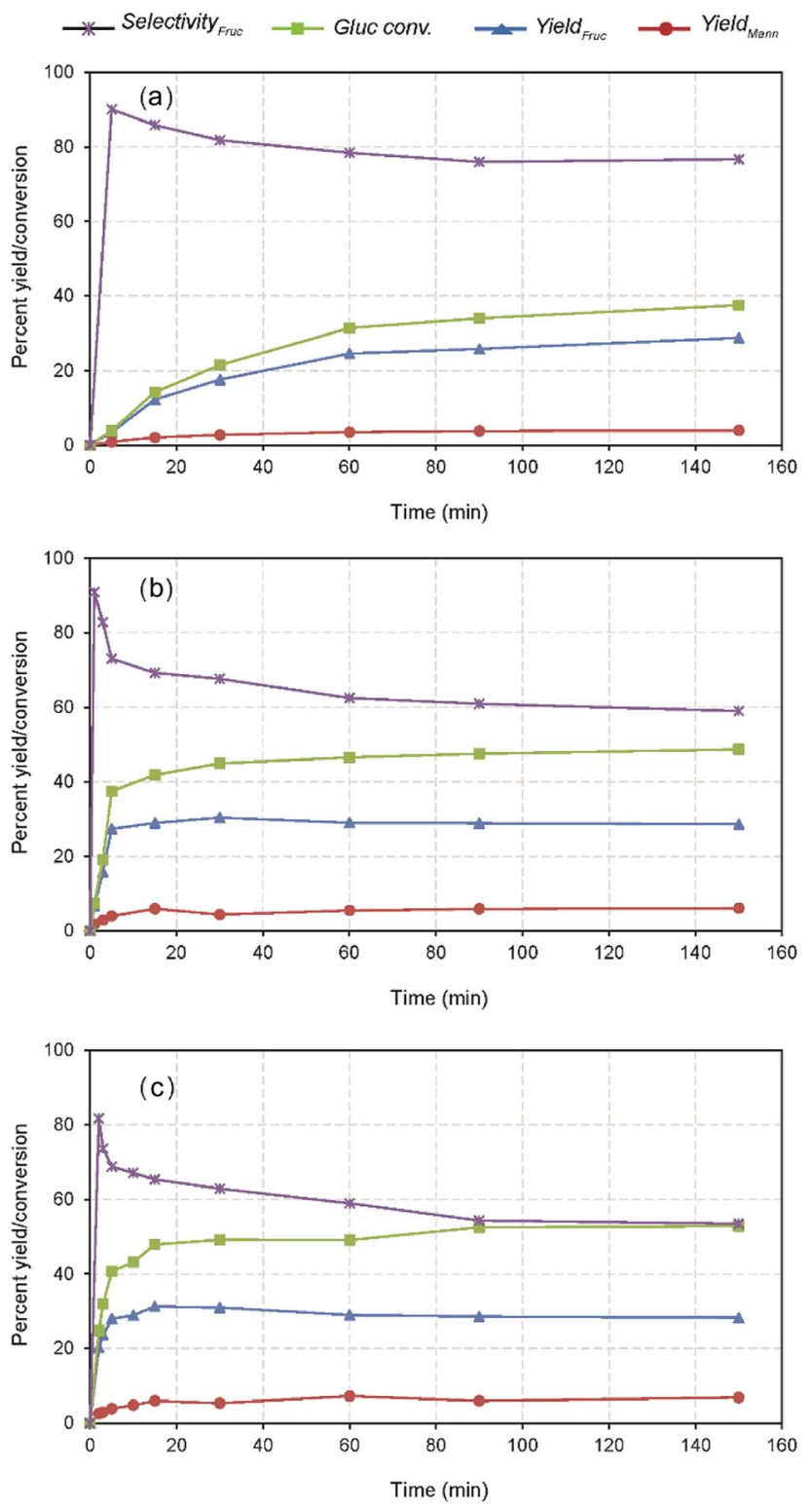

Fig. 4 Kinetics of glucose isomerization at different temperature conditions, (a) $60^{\circ} \mathrm{C}$, (b) $80^{\circ} \mathrm{C}$, and (c) $100{ }^{\circ} \mathrm{C}(10 \%$ wt catalyst dose on glucose).

with $76.75 \%$ fructose selectivity), respectively. This phenomenon explains that the reaction is controlled up to the specified times depending on the reaction severity and beyond, the activity practically reaches a plateau as displayed in Fig. 4. As mentioned earlier, the contrary responses achieved with the glucose conversion and fructose selectivity with all the temperatures are due to the carbohydrate degradation and reversible reactions. ${ }^{8}$ Mechanistically, the equilibrium constant for the glucose isomerization reaction lies $<0.6$, therefore, the reaction is reversible to form fructose and is unavoidable under the specified conditions. ${ }^{32}$ The temperature dependency of the GI reaction was verified using Arrhenius equation through the correlation plot obtained between $\ln K$ vs. 1/T (Fig. S6 in the ESI $\dagger$ ), outlining the increased catalytic activity of the reaction 
under raised temperature conditions $\left(R^{2}=0.99\right) .{ }^{22}$ The kinetic reaction rates also pronounced remarkable differences with respect to the temperatures $\left(60,80\right.$ and $\left.100^{\circ} \mathrm{C}\right)$, i.e., $1.0,6.0$ and $19.0 \times 10^{-4} \mathrm{~s}^{-1}$. The measured activation energy $\left(E_{\mathrm{a}}\right)$ of the reaction was $76 \mathrm{~kJ} \mathrm{~mol}^{-1}$, which is consistent with prior literature studies investigated under Lewis base condition in water system. ${ }^{36}$ The estimated carbon balance and TOF values were summarized in Table S1 in the ESI. $\dagger$ Although the increased productivity accomplished within the shorter residence time possibly ascribed to the enhanced surface reaction of nanotubes offering more basic sites and within mesopores, the stability of the catalyst was not satisfactory, resulting in a low glucose conversion with fructose yield after $15 \mathrm{~min}$. This suggests that higher catalyst deactivation and proximity to the thermodynamic equilibrium conversion for this reaction at $100{ }^{\circ} \mathrm{C}$ and higher reaction times. ${ }^{8}$ At last, it can be noticed that the post-reaction catalysts color turned from white to yellow, suggesting it contained a considerable amount of organic matter, based on the spectroscopic analysis ${ }^{23}$ (Fig. S7 in the ESI $\dagger$ ). At the same time, a proportionately increased mannose concentration with decreasing fructose yield with respect to time (after $15 \mathrm{~min}$ ) (Fig. 4). Suggesting that progression of reversible reaction through cis-enediol intermediate formation under favourable conditions due to the shift of reaction equilibrium, which results in producing a mixture of stereoisomers (i.e., mannose and glucose), ${ }^{37,38}$ as scheme depicted in Fig. 2 and S8 in the ESI. $\uparrow$ Nevertheless, the catalyst stimulated the maximum fructose productivity within relatively shorter residence time than the reported Lewis base catalysis, where slightly harsher conditions were employed (using more than $20 \%$ catalyst loading, $100{ }^{\circ} \mathrm{C}$, and $30 \mathrm{~min}$ ). ${ }^{8}$ Therefore, the plausible cause for this comparable yield achievement is due to leaching of $\mathrm{Na}^{+}$ions, and surface passivation by strongly adsorbed unwanted organic compounds led to catalyst deactivation. ${ }^{16}$

\section{Catalyst recycle-ability and regeneration}

In complementary, the nanotubes allowed easy recovery through the formal filtration and washing after the finish of the reaction. Solid catalysts reuse in heterogeneous catalysis is one of the most important advantages. However, the activity decay is the only in subject to without loss of their catalytic activity. ${ }^{22}$ In perception, formation and retention of carbonaceous materials (coke) inside pore structure, and leaching of ions from the solid material to the liquid product results in the deactivation of the solid catalysts during the reaction. ${ }^{39}$ In order to evaluate the recyclability of the recovered catalyst, consecutive repeat studies were conducted under the modest reaction conditions. The comparative results show (Fig. 5) that an adverse effect on glucose conversion (16\% relative lower to the fresh runs) and fructose yield (10\%) during recycling runs up to 4 was achieved when using the recovered nanotubes thermal treatment. This is supposed to be leaching of $\mathrm{Na}^{+}$ion, thereby reducing the basicity, blockage of pores due to the deposited unwanted carbonaceous matters, and agglomeration of catalyst particles. $^{22}$ In addition, results of the SEM analysis of both pre- and

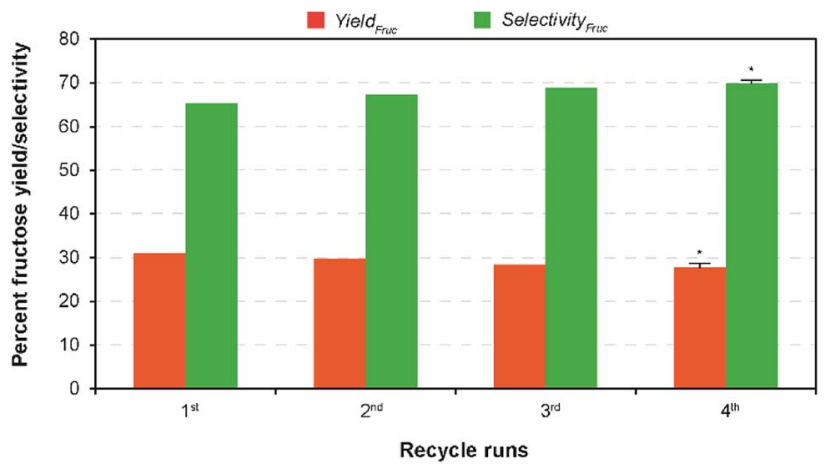

Fig. 5 Performance of recovered catalyst on each recycle runs of glucose isomerization conducted under modest reaction conditions (10\% wt catalyst dose at $100{ }^{\circ} \mathrm{C}$ for $\left.15 \mathrm{~min}\right)$. Error bar represents standard deviation, and asterisk (*) indicates significant at $p$-value $\leq$ 0.05 , respectively.

post-reaction catalysts clearly show the increased agglomeration of catalyst particles during the reaction that in turn cause a reduction in catalytic activity (Fig. S9 in the ESI†). Consequently, a simple regeneration technique involving $\mathrm{NaOH}$ was employed to the post-reaction catalyst solids after calcination under the similar conditions. The result shows that comparable fructose yield $(29.98 \% \mathrm{wt})$ with selectivity $(67.86 \%)$ was obtained under the modest reaction conditions to the maximum yield. This conveys that it is possible to regain its total catalytic activity through the conventional ion exchange protocol, demonstrating a cost-effective isomerization process. The results proposed that Na-TNT could be a promising candidature for the large-scale isomerization of glucose to fructose reaction through continuous process operations (most suitably fixed bed reactors).

In a typical GI reaction, it is postulated that stabilization of ions via hydroxylation of alkali metal oxide surface with the hydroxyl anions available in the solution (aqueous) initiates the reaction. ${ }^{34}$ In order to verify the mechanism, a series of comparative studies were conducted in alcohol systems, such as methanol $\left(\mathrm{p} K_{\mathrm{a}}=15.5\right)$, ethanol $\left(\mathrm{p} K_{\mathrm{a}}=15.9\right)$, and 1-propanol $\left(\mathrm{p} K_{\mathrm{a}}=16.1\right)$ medium (both diluted and non-diluted levels) under the modest conditions $\left(10 \%\right.$ wt catalyst dose at $100{ }^{\circ} \mathrm{C}$ for $30 \mathrm{~min}$ ) (Fig. 6). Since larger the substituents in alcohols are better electron donors, which destabilize the resulting $\mathrm{OH}^{-}$anions. The discouraging results show that effectiveness of the reaction was not up to the mark in alcohols, and no constructive correlation between the order of sequence of alcohols and yield results, predominantly, might be attributed to the combination of polarizability and solvation effects, ${ }^{40}$ and solubility of carbohydrate sugars. ${ }^{41}$ For instance, the systems achieved relatively $27-50 \%$ lower fructose yield $(2: 1$ alcohol to water ratio) than water system, whereas the diluted systems remarkably increased the fructose concentrations ( $\sim$-fold) accompanied by a better fructose selectivities $(\sim 90 \%)$. Nevertheless, the alcohol systems minimized the formation of byproducts and degradation products (Fig. S9 in the ESI $\dagger$ ). This suggests that Na-TNT is effective in the glucose to fructose conversion but only in $\mathrm{H}_{2} \mathrm{O}$ medium $\left(\mathrm{p} K_{\mathrm{a}}=14.0\right)$. 


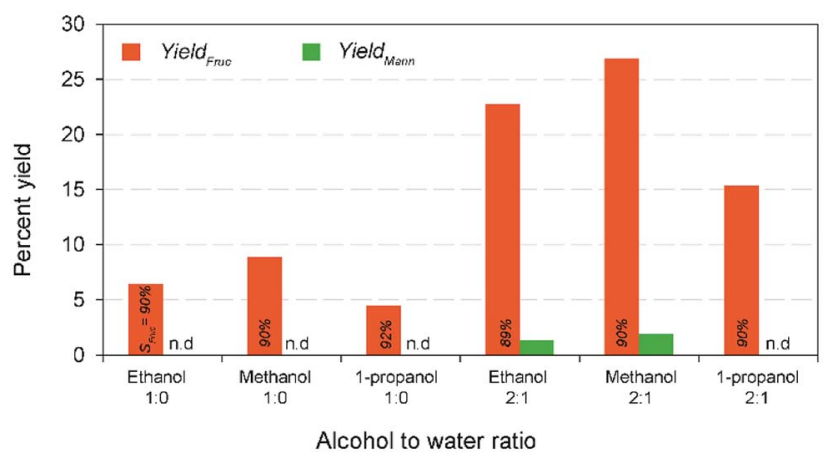

Fig. 6 Response result of glucose isomerization in different alcohol mediums at various dilution levels (at $100{ }^{\circ} \mathrm{C}$ for 15 min using $10 \% \mathrm{wt}$ catalyst dose). $S_{\text {Fruc }}$ - fructose selectivity. N.D. - not detected.

\section{Evaluation of cation exchanged-TNTs on glucose isomerization}

With regard to the catalyst basicity, the basic strength of the alkali-ion exchanged solid catalyst is rationalized by the negative charge of the oxygen atoms. Therefore, the base strength of TNT catalyst depends on the exchangeable alkali cations and it is reasonably increasing in the order of $\mathrm{Li}^{+}<$ $\mathrm{Na}^{+}<\mathrm{K}^{+}<\mathrm{Rb}^{+}<\mathrm{Cs}^{+}$within the monovalent alkali elements, according to the atomic properties (i.e., electrostatic interaction and hydrated radius). ${ }^{2,22}$ In order to evaluate the effectiveness of the other monovalent cation exchanged titanate nanotubes, namely Li-TNT, K-TNT and Cs-TNT on glucose to fructose isomerization, a comparative experiment was planned under the optimum processing conditions. The initial characterization of the as-synthesized catalysts revealed the maximum degree of ion exchange were $c a .56 \%$, $48 \%$, and $36 \%$, respectively. Although $\mathrm{Cs}^{+}$has the smallest hydrated radius within the group (226 pm), it can approach the surface the closest and be held the more tightly. These variations might be attributed to the relative affinity of a cation for a sorbent or the selectivity. In accordance with the literature studies that a reverse order is often observed with some hydrous oxides, for instance, the order of affinity of cations on titanium metal oxides is $\mathrm{Li}^{+}>\mathrm{Na}^{+}>\mathrm{Cs}^{+} .{ }^{42}$ The apparent isomerization results revealed (Fig. 7) that the fructose yield and selectivity varies depending on the basicity strength of the charge compensating ions. For example, $\mathrm{Li}^{+}$ exhibited a slightly lower fructose yield (29.97\%) with higher selectivity (68.89\%) when compared to the results achieved using $\mathrm{Na}^{+}$, whereas the others attained $16-22 \%$ relatively lower yields accompanied with a contrarily improved fructose selectivity (10-16\%). This is consistent with the ion exchange capacity of the elements $\left(\mathrm{K}^{+}\right.$and $\left.\mathrm{Cs}^{+}\right)$had comparatively lower degree value might be attributed to the increased hydrated radius in aqueous medium and other potential factors. ${ }^{43}$ Hence, even with the increase in basic nature with the ion-exchange of $\mathrm{Na}^{+}$on titanate nanotubes the poor activity was observed.

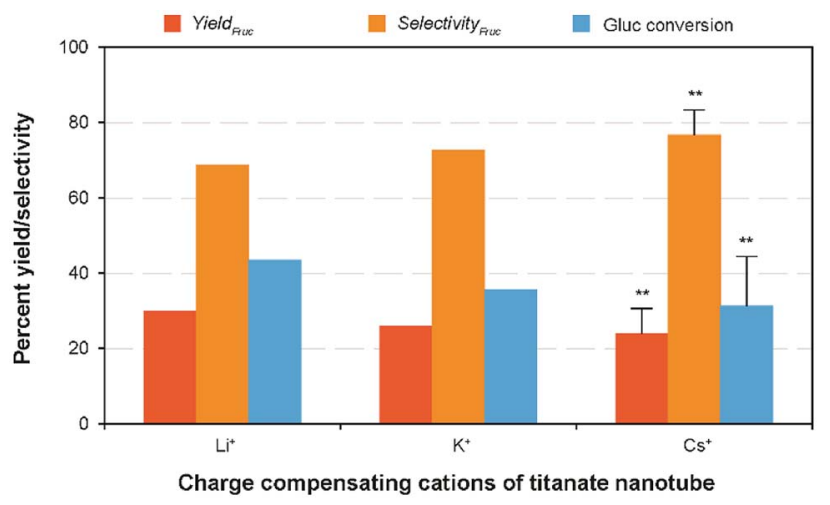

Fig. 7 Comparative result of glucose isomerization in the presence of various monovalent alkaline cations of titanate nanotubes under the optimum conditions. Error bar represents standard deviation, and asterisks $(*)$ and $(* *)$ indicate significant and non-significant at $p$-value $\leq 0.05$, respectively.

\section{Conclusions}

The present study demonstrated a single-step reaction of glucose isomerization to fructose over low-cost titanate material with $\mathrm{Na}^{+}$ions in aqueous media. In a breakthrough, typical heterogeneous Lewis base catalysis expedites maximum fructose yield (31.26\%) with higher selectivity (65.26\%) within 15 min or less, establishing a cost-effective process methodology. Presumably, the increased basic sites due to the enhanced surface morphology of the catalyst (titanate nanotube) greatly influenced the catalytic reaction, thereby enabling higher glucose conversion. Na-TNTs exhibited a satisfactory performance over glucose isomerization to fructose reaction among the nanotubes tested consisting of $\mathrm{Li}^{+}, \mathrm{K}^{+}$, and $\mathrm{Cs}^{+}$, suggesting it could serve as a potential catalyst for large-scale with recyclable advantages. Though the fast leaching of $\mathrm{Na}^{+}$ ions during reaction impedes the fructose productivity, it is possible to regain its activity and durability through a simple regeneration protocol involving only $\mathrm{NaOH}$.

\section{Conflicts of interest}

There are no conflicts to declare.

\section{Acknowledgements}

The authors gratefully thank Department of Biotechnology (Government of India), New Delhi, India for their consistent financial support. S. Elumalai acknowledges Department of Science and Technology (DST-SERB), New Delhi for the financial support through Grant No. YSS/2014/000031.

\section{Notes and references}

1 M. J. Climent, A. Corma and S. Iborra, Green Chem., 2014, 16, 516-547.

2 I. Delidovich and R. Palkovits, ChemSusChem, 2016, 9, 547561. 
3 H. Li, S. Yang, S. Saravanamurugan and A. Riisager, ACS Catal., 2017, 7, 3010-3029.

4 Y. Román-Leshkov, M. Moliner, J. A. Labinger and M. E. Davis, Angew. Chem., Int. Ed., 2010, 49, 8954-8957.

5 A. Norton, H. Nguyen, N. L. Xiao and D. G. Vlachos, RSC Adv., 2018, 8, 17101-17109.

6 Z. Xue, M.-G. Ma, Z. Li and T. Mu, RSC Adv., 2016, 6, 9887498892.

7 M. Moliner, Y. Román-Leshkov and M. E. Davis, Proc. Natl. Acad. Sci. U. S. A., 2010, 107, 6164-6168.

8 I. Graça, D. Iruretagoyena and D. Chadwick, Appl. Catal., B, 2017, 206, 434-443.

9 S. Li, T. Josephson, D. G. Vlachos and S. Caratzoulas, J. Catal., 2017, 355, 11-16.

10 Z. Zhu, H. Xu, J. Jiang, Y. Guan and P. Wu, J. Catal., 2017, 352, 1-12.

11 M. Watanabe, Y. Aizawa, T. Iida, R. Nishimura and H. Inomata, Appl. Catal., A, 2005, 295, 150-156.

12 S. Yu, E. Kim, S. Park, I. K. Song and J. C. Jung, Catal. Commun., 2012, 29, 63-67.

13 S. Lima, A. S. Dias, Z. Lin, P. Brandão, P. Ferreira, M. Pillinger, J. Rocha, V. Calvino-Casilda and A. A. Valente, Appl. Catal., A, 2008, 339, 21-27.

14 Q. Yang, S. Zhou and T. Runge, J. Catal., 2015, 330, 474-484.

15 Y. Ono and T. Baba, Catal. Today, 1997, 38, 321-337.

16 J. Faria, M. Pilar Ruiz and D. E. Resasco, ACS Catal., 2015, 5, 4761-4771.

17 M. Kitano, K. Nakajima, J. N. Kondo, S. Hayashi and M. Hara, J. Am. Chem. Soc., 2010, 132, 6622-6623.

18 L. L. Marciniuk, P. Hammer, H. O. Pastore, U. Schuchardt and D. Cardoso, Fuel, 2014, 118, 48-54.

19 M. Watanabe, Y. Aizawa, T. Iida, T. M. Aida, C. Levy, K. Sue and H. Inomata, Carbohydr. Res., 2005, 340, 1925-1930.

20 G. Martra, Appl. Catal., A, 2000, 200, 275-285.

21 C. Lanziano, F. Rodriguez, S. Rabelo, R. Guirardello, V. Da Silva and C. Rodell, Chem. Eng. Trans., 2014, 37, 589-594.

22 C. Moreau, R. Durand, A. Roux and D. Tichit, Appl. Catal., A, 2000, 193, 257-264.

23 T. Kasuga, M. Hiramatsu, A. Hoson, T. Sekino and K. Niihara, Langmuir, 1998, 14, 3160-3163.

24 D. Nepak and S. Darbha, Catal. Commun., 2015, 58, 149-153.
25 A. Gajović, I. Friščić, M. Plodinec and D. Iveković, J. Mol. Struct., 2009, 924, 183-191.

26 N. Liu, X. Chen, J. Zhang and J. W. Schwank, Catal. Today, 2014, 225, 34-51.

27 Y. R. Smith, R. S. Ray, K. Carlson, B. Sarma and M. Misra, Materials, 2013, 6, 2892-2957.

28 X. Sun, X. Chen and Y. Li, Inorg. Chem., 2002, 41, 4996-4998.

29 F. Cavani, C. Felloni, D. Scagliarini, A. Tubertini, C. Flego and C. Perego, Stud. Surf. Sci. Catal., 2000, 143, 953-961.

30 V. Štengl, S. Bakardjieva, J. Šubrt, E. Večerníková, L. Szatmary, M. Klementová and V. Balek, Appl. Catal., B, 2006, 63, 20-30.

31 C. M. Rodrigues, O. P. Ferreira and O. L. Alves, J. Braz. Chem. Soc., 2010, 21, 1341-1348.

32 J. M. Carraher, C. N. Fleitman and J.-P. Tessonnier, ACS Catal., 2015, 5, 3162-3173.

33 C. Liu, J. M. Carraher, J. L. Swedberg, C. R. Herndon, C. N. Fleitman and J.-P. Tessonnier, ACS Catal., 2014, 4, 4295-4298.

34 A. A. Marianou, C. M. Michailof, A. Pineda, E. F. Iliopoulou, K. S. Triantafyllidis and A. A. Lappas, ChemCatChem, 2016, 8, 1100-1110.

35 V. Galstyan, E. Comini, G. Faglia and G. Sberveglieri, Sensors, 2013, 13, 14813-14838.

36 Q. Yang, M. Sherbahn and T. Runge, ACS Sustainable Chem. Eng., 2016, 4, 3526-3534.

37 Y. J. Topper and D. Stetten, J. Biol. Chem., 1951, 189, 191-202.

38 S. J. Angyal, in Glycoscience: Epimerisation, Isomerisation and Rearrangement Reactions of Carbohydrates, ed. A. E. Stütz, Springer, Berlin, Heidelberg, 2001, pp. 1-14.

39 M. D. Argyle and C. H. Bartholomew, Catalysts, 2015, 5, 145269.

40 Y. Zeng, X. Chen, D. Zhao, H. Li, Y. Zhang and X. Xiao, Fluid Phase Equilib., 2012, 313, 148-155.

41 L. A. Alves, J. B. Almeida e Silva and M. Giulietti, J. Chem. Eng. Data, 2007, 52, 2166-2170.

42 Y. Berube and P. L. de Bruyn, J. Colloid Interface Sci., 1968, 27, 305-318.

43 S. Despax, B. Estrine, N. Hoffmann, J. Le Bras, S. Marinkovic and J. Muzart, Catal. Commun., 2013, 39, 35-38. 